\title{
Simultaneous Administration of Adjuvant Donor Bone Marrow in Pancreas Transplant Recipients
}

\author{
Robert J. Corry, MD, Pradip K. Chakrabarti, MD, Ron Shapiro, MD, Abdul S. Rao, MD, DPhil, lgor Dvorchik, PhD, \\ Mark L. Jordan, MD, Velma P. Scantlebury, MD, Carlos A. Vivas, MD, John J. Fung, MD, PhD, and Thomas E. Starzl, MD, PhD
}

From the Thomas E. Starzl Transplantation Institute, Department of Surgery, University of Pittsburgh School of Medicine, Pittsburgh, Pennsylvania

\section{Objective}

The effect of donor bone marrow was evaluated for its potentially favorable effect in the authors' simultaneous pancreas/ kidney transplant program.

\section{Methods \\ From July 1994 to January 1999, 177 pancreas transplants were performed, 151 of which were simultaneous pancreas/ kidney transplants. All patients received tacrolimus, mycophe- nolate mofetil, and steroids for immunosuppression (azathio- prine was used in the first year of the program). Fifty-three simultaneous pancreas/kidney transplant recipients received perioperative unmodified donor bone marrow, 3 to $6 \times 10^{8}$ cells/kg.}

\section{Results}

Overall actuarial survival rates at 1 and 3 years were $98 \%$ and 95\% (patient), $95 \%$ and $87 \%$ (kidney), and $86 \%$ and $80 \%$ (pancreas), respectively. In the adjuvant bone marrow group, 1 - and 3-year survival rates were $96 \%$ and $91 \%$ (patient), $95 \%$ and $87 \%$ (kidney), and $83 \%$ and $83 \%$ (pancreas), respectively. For 98 recipients who did not receive bone marrow, survival rates at 1 and 3 years were 100\% and 98\% (patient), $96 \%$ and $86 \%$ (kidney), and $87 \%$ and $79 \%$ (pancreas), respectively. No pancreas allografts were lost after 3 months in bone marrow recipients, and seven in the non-bone marrow recipients were lost to rejection at $0.7,6.7,8.8,14.6$, 24.1, 24.3, and 25.5 months.

Twenty-two percent of bone marrow patients were steroidfree at 1 year, $45 \%$ at 2 years, and $67 \%$ at 3 years. Nineteen percent of the non-bone marrow recipients were steroid-free at 1 year, $38 \%$ at 2 years, and $45 \%(p=0.02)$ at 3 years. The mean acute cellular rejection rate was $0.94 \pm 1.1$ in the bone marrow group and $1.57 \pm 1.3(p=0.003)$ in the non-bone marrow group (includes borderline rejection and multiple rejections). The level of donor cell chimerism in the peripheral blood of bone marrow patients was at least two logs higher than in controls.

\section{Conclusion}

In this series, which represents the largest experience with adjuvant bone marrow infusion in pancreas recipients, there was a higher steroid withdrawal rate $(p=0.02)$, fewer rejection episodes, and no pancreas graft loss after 3 months in bone marrow recipients compared with contemporaneous controls. All pancreas allografts lost to chronic rejection ( $\mathrm{n}=$ 6) were in the non-bone marrow group. Donor bone marrow administered around the time of surgery may have a protective effect in pancreas transplantation.
Over the past 10 years. the evolution of pancreas transplantation has been impressive. Outcomes are being achieved that match those of the other solid organ transplants in terms of patient and graft survival rates. ${ }^{1.2}$ reduc-

Presented at the 119th Annual Meeting of the American Surgical Association. April 15-17. 1999. Hyatt Regency Hotel. San Diego. California. Supported by NIH Grant No. Al38899.

Correspondence: Robert J. Corry. MD. 4-Falk Clinic. 3601 Fifth Ave.. Pittsburgh. PA 15213.

Accepted for publication April 1999. tion in the surgical complication rate,,$^{3,4}$ and improvement in quality of life. ${ }^{5.6}$ In addition, there is emerging evidence that the secondary complications of diabetes can be stabilized and even reversed with prolonged normal carbohydrate metabolism. ${ }^{7-9}$ The principal reasons for the improved longterm success have been both the increasing experience with tacrolimus-based immunosuppression and the reduction in surgical complications, in part associated with the reintroduction of primary enteric drainage of the exocrine secretions. $^{10.11}$ Complications associated with duodenocystostomy, the most common method of exocrine drainage, have 
Table 1. RECIPIENT DEMOGRAPHICS

\begin{tabular}{|c|c|c|c|}
\hline & $\begin{array}{c}\text { All SPK } \\
(n=151)\end{array}$ & $\begin{array}{c}\text { Bone } \\
\text { Marrow } \\
(n=53)\end{array}$ & $\begin{array}{c}\text { No Bone } \\
\text { Marrow } \\
(n=98)\end{array}$ \\
\hline \multicolumn{4}{|l|}{ Age (yrs) } \\
\hline Mean \pm SD & $40.2 \pm 7$ & $39.8 \pm 8.6$ & $40.0 \pm 6.9$ \\
\hline Range & $27.6-57$ & $27.6-56.1$ & $28.8-57$ \\
\hline \multicolumn{4}{|l|}{ Sex } \\
\hline Male & $53 \%$ & $51 \%$ & $56 \%$ \\
\hline Female & $47 \%$ & $49 \%$ & $44 \%$ \\
\hline \multicolumn{4}{|l|}{ Race } \\
\hline African American & $7 \%$ & $10 \%$ & $4 \%$ \\
\hline White & $93 \%$ & $90 \%$ & $96 \%$ \\
\hline $\mathrm{PRA}(\% \pm S D)$ & $3 \pm 7.5$ & $2.7 \pm 4.7$ & $3.2 \pm 8.6$ \\
\hline \multicolumn{4}{|l|}{ Drainage } \\
\hline Enteric & $88 \%$ & $85 \%$ & $90 \%$ \\
\hline Bladder & $12 \%$ & $15 \%$ & $10 \%$ \\
\hline \multicolumn{4}{|l|}{ Pretransplant dialysis } \\
\hline None & $35 \%$ & $30 \%$ & $37 \%$ \\
\hline Peritoneal & $23 \%$ & $24 \%$ & $22 \%$ \\
\hline Hemodialysis & $42 \%$ & $46 \%$ & $41 \%$ \\
\hline
\end{tabular}

included fistula, urinary tract infection and sepsis, and metabolic acidosis, and have required conversion to enteric drainage in $25 \%$ of pancreas recipients. ${ }^{1}$

Despite these improving outcomes, graft loss to rejection still occurs in close to $10 \%$ of patients, as reported by Sollinger et al in an excellent large series of combined pancreas/kidney recipients. ${ }^{1}$ If a significant reduction in rejection-related graft loss could be achieved, even more patients would benefit from the procedure.

The tolerance induction model, using adjuvant perioperative donor-specific bone marrow, was based on the obser-

Table 2. DONOR DEMOGRAPHICS

\begin{tabular}{|c|c|c|c|}
\hline & $\begin{array}{l}\text { All SPK } \\
(n=151)\end{array}$ & $\begin{array}{c}\text { Bone } \\
\text { Marrow } \\
(n=53)\end{array}$ & $\begin{array}{l}\text { No Bone } \\
\text { Marrow } \\
(n=98)\end{array}$ \\
\hline \multicolumn{4}{|l|}{ Age (yrs) } \\
\hline Mean $\pm S D$ & $30.2 \pm 13.6$ & $27.6 \pm 12.8$ & $31.6 \pm 13.8$ \\
\hline Range & $6.8-61.4$ & $10.9-55.5$ & $6.8-61.4$ \\
\hline \multicolumn{4}{|l|}{ Sex } \\
\hline Male & $51 \%$ & $60 \%$ & $45 \%$ \\
\hline Female & $49 \%$ & $40 \%$ & $55 \%$ \\
\hline \multicolumn{4}{|l|}{ Race } \\
\hline White & $85 \%$ & $84 \%$ & $86 \%$ \\
\hline African American & $8 \%$ & $7 \%$ & $9 \%$ \\
\hline Other & $6 \%$ & $9 \%$ & $5 \%$ \\
\hline \multicolumn{4}{|l|}{$\begin{array}{r}\text { Mean cold ischemia } \\
\text { time } \pm S D(h r s)\end{array}$} \\
\hline Kidney & $16.3 \pm 4.6$ & $15.8 \pm 5$ & $16.5 \pm 4$ \\
\hline Pancreas & $17.7 \pm 4$ & $17.5 \pm 4$ & $18 \pm 3.8$ \\
\hline
\end{tabular}

Table 3. HISTOCOMPATIBILITY MATCHING

\begin{tabular}{ccc}
\hline $\begin{array}{c}\text { Antigens } \\
\text { Matched }\end{array}$ & $\begin{array}{c}\text { Bone Marrow } \\
\text { (n = 53) }\end{array}$ & $\begin{array}{c}\text { No Bone Marrow } \\
\text { (n = 98) }\end{array}$ \\
\hline 0 & $25 \%$ & $30 \%$ \\
1 & $42 \%$ & $32 \%$ \\
2 & $24 \%$ & $24 \%$ \\
3 & $5 \%$ & $8 \%$ \\
4 & $4 \%$ & $5 \%$ \\
5 & - & - \\
6 & - & $1 \%$ \\
\hline
\end{tabular}

vation by Starzl et al that donor leukocytes were found in organs and tissues of long-term surviving transplant recipients. ${ }^{12}$ This microchimeric state, found many years after transplantation in successfully transplanted patients, some of whom were no longer receiving immunosuppression, suggested a favorable balance of the natural host-versusgraft and graft-versus-host reactions. Several reports since have confirmed an augmentation of chimerism in transplanted recipients who were simultaneously given donor bone marrow infusions. ${ }^{13.14}$ This report will describe in detail, for the first time, our observations in a series of simultaneous pancreas/kidney transplant recipients, transplanted between July 1994 and January 1999, who either received or did not receive perioperative donor bone marrow, mainly as a function of the donor family's giving permission for removal of the vertebral bodies.

\section{MATERIALS AND METHODS}

\section{Recipient Selection and Demographics}

Recipients were evaluated by a transplant surgeon, a transplant internist, a nurse transplant coordinator, and other members of the health care team. All patients had type I diabetes and ranged in age from 27.6 to 57 years. All patients underwent a dobutamine echocardiogram or an adenosine thallium scan, which was reviewed by a cardiologist to seek evidence of ischemia and to determine whether a cardiac catheterization was needed. If a potentially correctable lesion appeared on cardiac catheterization, intervention was carried out before listing the patient for transplantation. A few patients with uncorrectable and critical coronary disease were not listed because the risk was thought to outweigh the benefit. No other exclusion criteria were used, including amputations, blindness, or age older than 55. Demographics showed a nearly equal percentage of characteristics in the bone marrow and non-bone marrow groups (Table 1).

\section{Donor Selection and Characteristics}

All donors younger than age 65 were considered. Ages ranged from 6.8 to 61.4 years. with a mean age of 30.3 


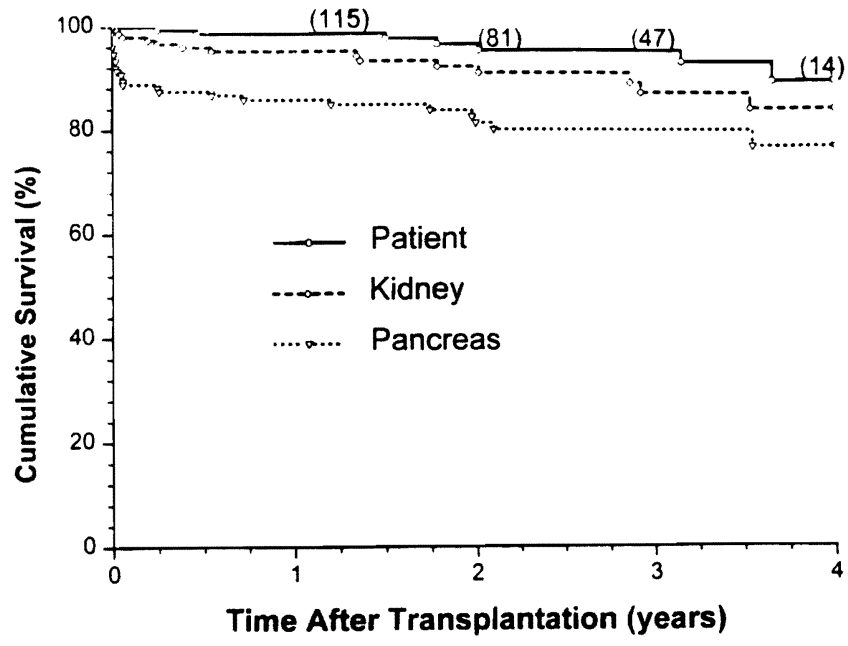

Figure 1. Overall patient, kidney, and pancreas allograft survival for 151 consecutive simultaneous pancreas/kidney transplants.

years. Donors with a history of cardiac arrest, two or more vasopressors, or hemodynamic instability were not excluded unless there was evidence of progressive renal dysfunction or pancreatic ischemia with a rising serum lipase determination.

Acceptance criteria and data regarding the use of "highrisk" donors were described in a recent report. ${ }^{15}$ The results of these high-risk donor pancreases, once transplanted, were not different from the overall results, provided that the organs were carefully selected based on the extent of fatty infiltration and fibrosis and the adequacy of the back-table flush with University of Wisconsin solution (Viospan, DuPont, Wilmington, DE). Donor demographics are detailed in Table 2. Histocompatibility matching is shown in Table 3.

The donor operation was a rapid en bloc liver and pancreas removal with separation of the two organs at the donor hospital: the technical details have been reported earlier. ${ }^{16}$ The rapid en bloc technique was applicable for hemodynamically unstable donors and donors without a heart beat as well as stable donors.

The final selection of an organ from a high-risk donor was based on careful inspection of the pancreas at the time

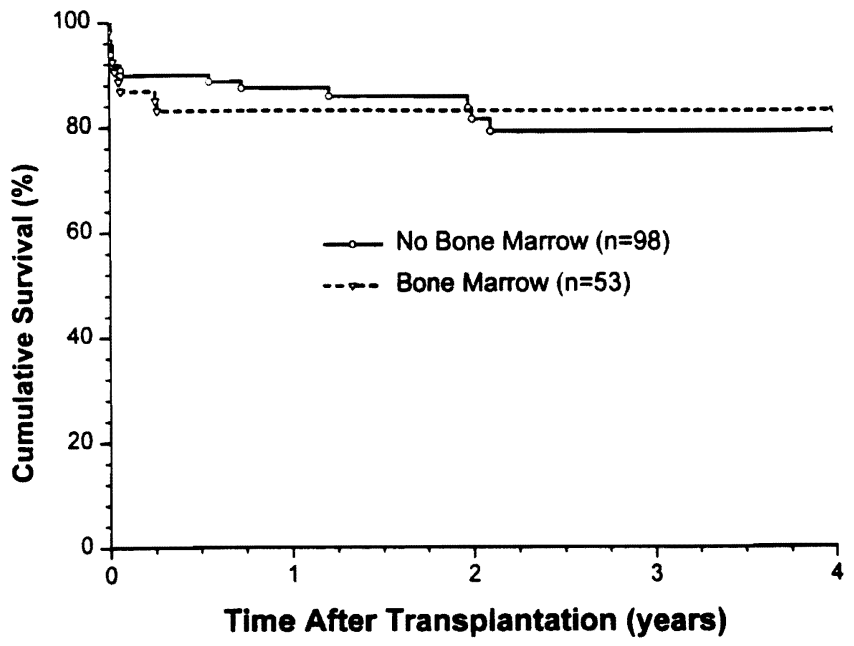

Figure 2. Pancreas allograft survival, comparing adjuvant bone marrow (BM) vs. no BM.

of back-table preparation, particularly with regard to the appearance of the pancreatic parenchyma; the quality of the vessels, including the $\mathrm{Y}$ graft; and the rate of venous effluent at the time of back-table flush.

\section{Transplant Procedure}

The kidney transplant was usually performed first. Kidneys were placed in a retroperitoneal position on the left side through a lower abdominal oblique incision, which was closed before the pancreas procedure was started.

The pancreas was transplanted through a separate right lower quadrant intraperitoneal oblique incision. During the kidney transplant procedure, or before both procedures, the bench work on the pancreas was performed. This included individual ligation of the mesenteric vessels at the root of the mesentery, shortening of the duodenum to a $7-$ to $10-\mathrm{cm}$ segment, ligation of the common bile duct, and joining of the donor iliac $\mathrm{Y}$ graft to the splenic and superior mesenteric arteries. The donor common iliac vein graft was used on several occasions to lengthen the portal vein, if it had been

Table 4. CAUSES OF PANCREAS FAILURES IN 151 SPK PATIENTS

\begin{tabular}{|c|c|c|c|c|c|}
\hline No. & Causes & $\begin{array}{c}\text { Mean Survival in Days } \\
\text { (Range) }\end{array}$ & BM & No BM & p Value \\
\hline 1 & Thrombosis $(n=8)$ & $1(0-2)$ & 2 & 6 & NS \\
\hline 2 & Pancreatitis ( $n=7$ ) & $11(7-21)$ & 5 & 2 & NS \\
\hline 3 & Rejection (n $=7$ ) & 342 (12-729) & - & 7 & 0.03 \\
\hline 4 & Death $(n=2)$ & $373(92-655)$ & 2 & - & NS \\
\hline 5 & Sepsis $(n=2)$ & $58(21-96)$ & 1 & 1 & NS \\
\hline 6 & Unknown $(n=1)$ & 1295 & 1 & - & NS \\
\hline
\end{tabular}




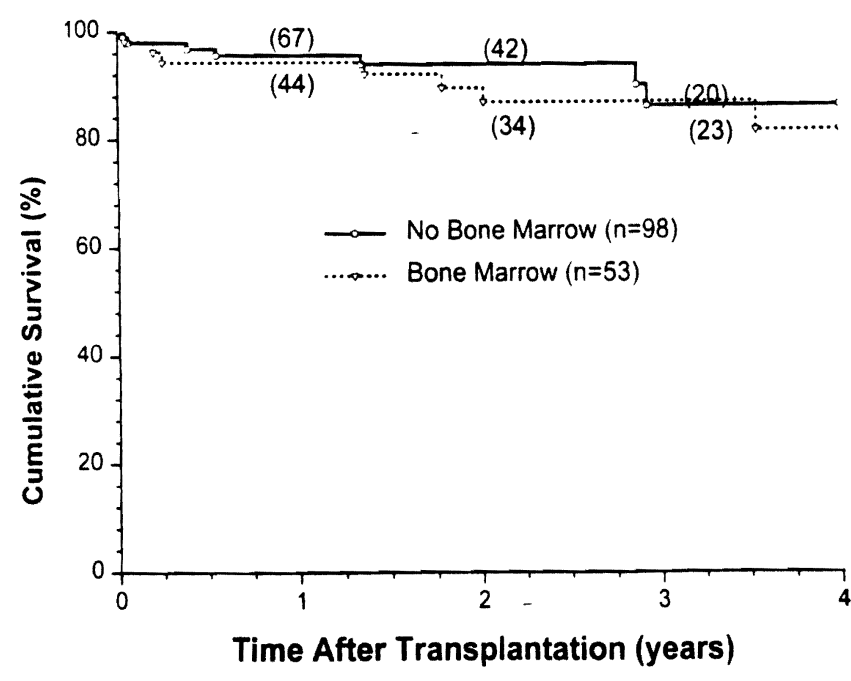

Figure 3. Kidney allograft survival, comparing adjuvant bone marrow (BM) vs. no BM.

divided near the junction of the splenic vein and the superior mesenteric vein.

The transplant procedure was performed by suturing the portal vein, with or without an extension graft, to either the external or common iliac vein and the donor Y graft common iliac artery to the recipient external, common, or internal iliac artery, depending on the positioning of the organ and the quality of the recipient vessels. The end of the duodenum was joined to the side of the proximal jejunum in a standard two-layer anastomosis. With the bladder-drainage technique, the organ was placed lower on the iliac vessels and a two-layer side-to-side duodenocystostomy was performed. ${ }^{17}$

\section{Immunosuppression}

From the beginning of the program in July 1994, the immunosuppressive protocol consisted of a triple-drug regimen based on tacrolimus. A loading dose of oral tacrolimus, $0.15 \mathrm{mg} / \mathrm{kg}$, was given on admission before transplantation, and $500 \mathrm{mg}$ intravenous methylprednisolone was administered during surgery after induction of anesthesia. After surgery, the patients were maintained on intravenous tacrolimus at a constant infusion for 4 to 7 days to maintain

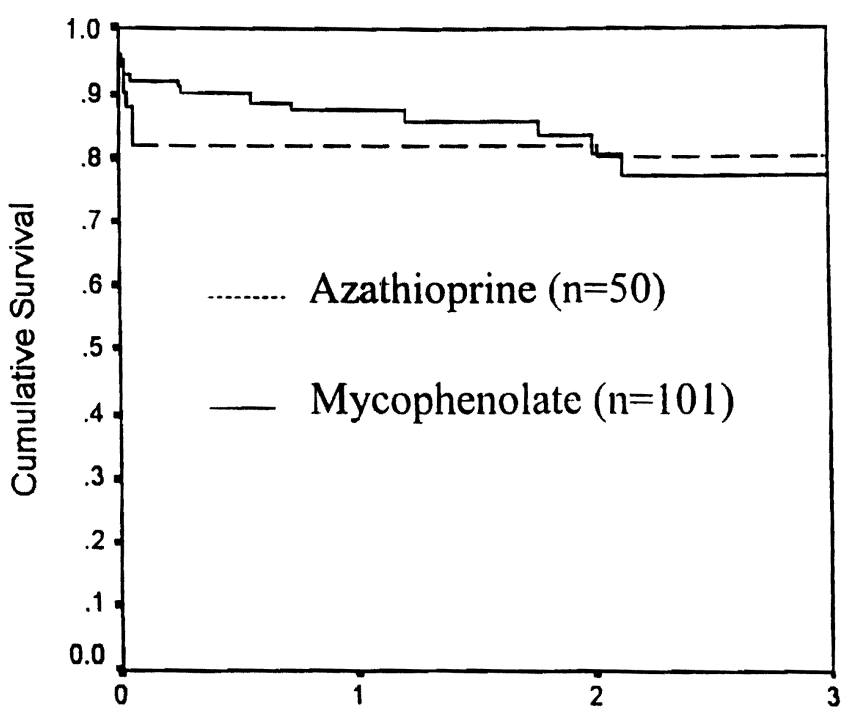

Survival after Transplantation (years)

Figure 4. Pancreas allograft survival with azathioprine vs. mycophenolate as a third immunosuppressive agent.

whole blood levels of 20 to $25 \mathrm{ng} / \mathrm{ml}$ for the first 2 weeks. Once the patients could tolerate a diet, they were converted to oral tacrolimus to maintain target whole blood trough levels of 15 to $20 \mathrm{ng} / \mathrm{ml}$ by 1 month, 10 to $15 \mathrm{ng} / \mathrm{ml}$ by 3 months, and 5 to $12 \mathrm{ng} / \mathrm{ml}$ chronically. Mycophenolate mofetil, $1 \mathrm{~g}$ twice a day, was given after surgery, with a dose reduction if the drug was not tolerated because of diarrhea or other gastrointestinal tract symptoms. Only a few patients were converted to azathioprine. All patients received tapering steroid doses after surgery, starting at $200 \mathrm{mg} /$ day and reduced by $40 \mathrm{mg} /$ day to $20 \mathrm{mg} /$ day by day 6 . If tolerated. prednisone was slowly tapered over the first 2 years, with discontinuation of the drug in $>60 \%$ of patients.

Rejection was treated with steroids in all cases, either intravenous methylprednisolone boluses for 3 days or a single methylprednisolone bolus followed by the steroid recycle, as described above. A few patients received antilymphocyte therapy if the rejections were not responsive to steroids. The tirst 50 patients received azathioprine at 1.5 to $2 \mathrm{mg} / \mathrm{kg}$ at the beginning of the program, before mycophenolate was available. Only three patients were converted

Table 5. CAUSES OF KIDNEY FAILURE IN 151 SPK PATIENTS

\begin{tabular}{|c|c|c|c|c|c|}
\hline No. & Causes & $\begin{array}{l}\text { Mean Survival in Days } \\
\text { (Range) }\end{array}$ & $\mathbf{B M}$ & No BM & p Value \\
\hline 1 & Rejection & $609(12-1291)$ & $2 *$ & 6 & NS \\
\hline 2 & Death $(n=4)$ & 509 (92-739) & 3 & 1 & NS \\
\hline 3 & Sepsis $(n=2)$ & $58(21-96)$ & 2 & - & NS \\
\hline 4 & Thrombosis $(n=1)$ & 21 & - & 1 & NS \\
\hline
\end{tabular}




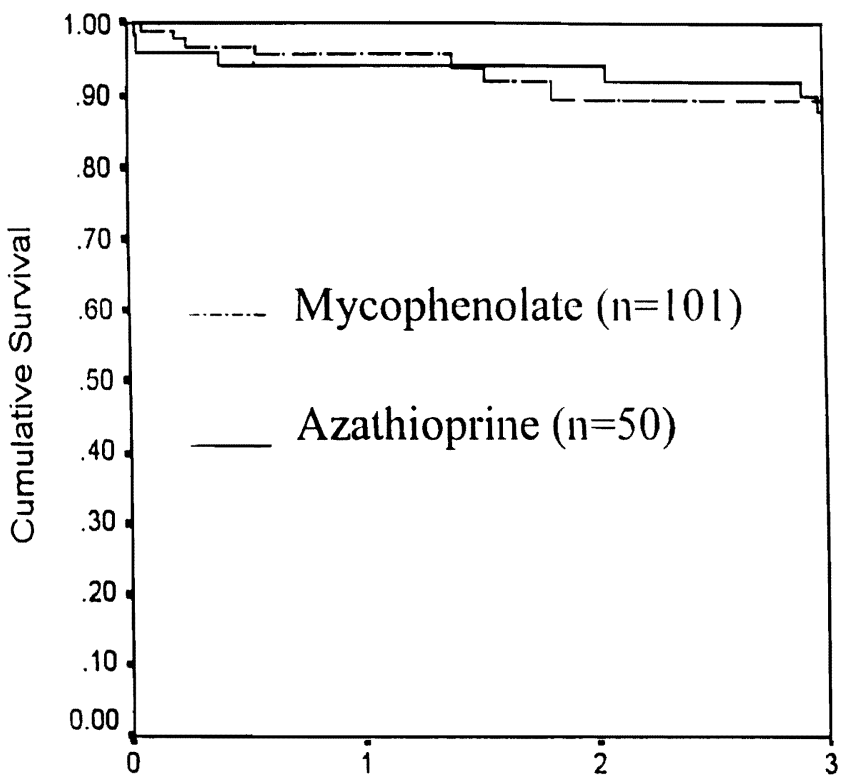

Survival after Transplantation (years)

Figure 5. Kidney allograft survival with azathioprine vs. mycophenolate as a third immunosuppressive agent.

from tacrolimus to cyclosporine because of persistent hyperglycemia that did not respond to dose reduction.

\section{Isolation and Infusion of Donor Bone Marrow With In Vitro Monitoring}

Bone marrow cells were harvested from the vertebral bodies of the cadaveric donors by a method described previously. ${ }^{18}$ In patients receiving perioperative donor bone marrow after organ revascularization, 3 to $6 \times 10^{8}$ unmodified cells per kilogram was infused through a central intra-

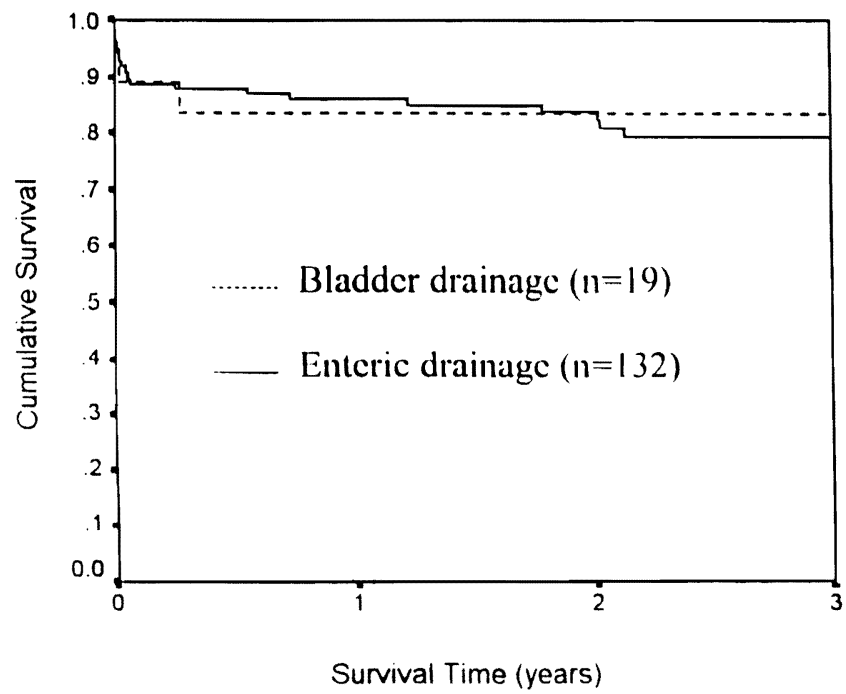

Figure 6. Panoreas allograft survival in simultaneous pancreas/kidney iransplants. comparing bladder vs. enteric drainage.

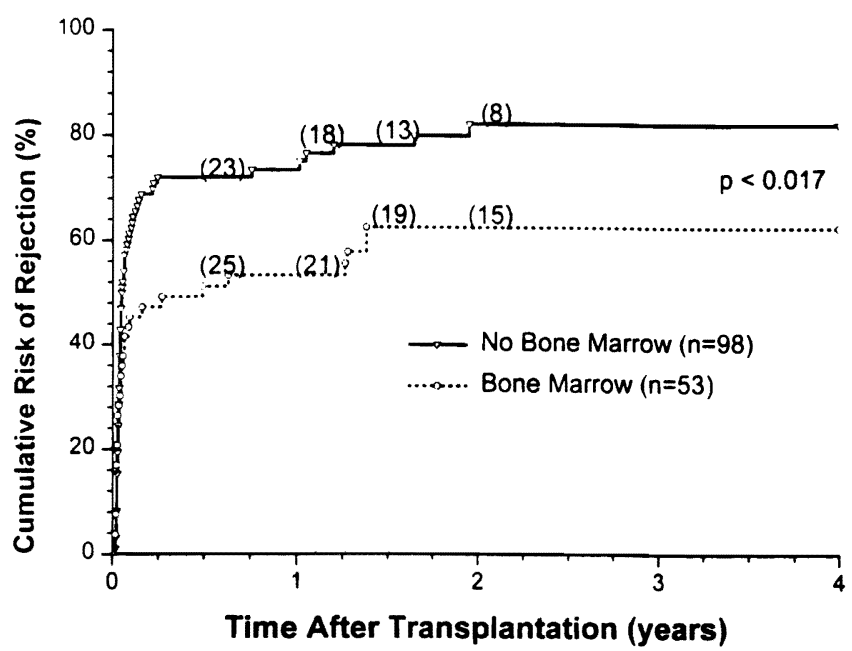

Figure 7. Cumulative risk of rejection in simultaneous pancreas/kidney transplants, comparing adjuvant bone marrow (BM) vs. no BM administration.

venous line. Using a quantitative method of nested polymerase chain reaction, ${ }^{19}$ the presence of donor cell chimerism was serially determined in the peripheral blood of both the study and control patients.

\section{Statistical Methods}

Data were recorded as mean \pm SD. Time-dependent variables-allograft failure and death-were estimated by Kaplan-Meier survival analysis. Comparison of rate of rejection in the bone marrow versus non-bone marrow groups was performed by log-rank analysis. Comparison of means between independent groups of continuous variables was estimated by independent sample $t$ test. Pearson chi square test or Fisher's exact test when appropriate was done for categorical data. Probability values $<0.05$ were considered significant.

\section{Ethical Considerations}

All subjects reported in this manuscript were thoroughly informed at the time of the transplant evaluation about the

Table 6. REJECTION ANALYSIS*

\begin{tabular}{lccc}
\hline $\begin{array}{c}\text { No. of Rejection } \\
\text { Episodes }\end{array}$ & $\begin{array}{c}\text { BM } \\
\text { (n=53) }\end{array}$ & $\begin{array}{c}\text { No BM } \\
\text { (n= }=\mathbf{9 8})\end{array}$ & p Value \\
\hline None & $23(43 \%)$ & $21(21 \%)$ & 0.05 \\
One & $18(34 \%)$ & $34(34 \%)$ & NS \\
Two or more & $12(22 \%)$ & $43(44 \%)$ & 0.05 \\
Mean & $0.94 \pm 1.1$ & $1.57 \pm 1.3$ & 0.003
\end{tabular}

- Cox regression analvsis tor proportional hazara assumption BM vs. no BM. nazard at $0.59(p=0.017)$

$\mathrm{BM}$. bone marrow. 
Table 7. PERCENTAGE OF PATIENTS STEROID-FREE

\begin{tabular}{cccc}
\hline Steroid-Free & BM & No BM & p Value \\
\hline Year 1 & $22 \%$ & $19 \%$ & NS \\
Year 2 & $45 \%$ & $38 \%$ & NS \\
Year 3 & $67 \%$ & $45 \%$ & 0.02
\end{tabular}

BM, bone marrow.

bone marrow augmentation study, in accord with the ethical standards of the Internal Review Board of the University of Pittsburgh, and gave their written consent before the transplant procedure.

\section{RESULTS}

The overall patient. kidney, and pancreas actuarial survival rates in 151 patients are shown in Figure 1, with 3-year actuarial survival rates of $95 \%, 87 \%$, and $80 \%$, respectively. Figure 2 shows the pancreas Kaplan-Meier survival curves: no pancreases were lost after 3 months in recipients who received simultaneous donor bone marrow, whereas seven pancreases were lost to rejection in patients who did not receive donor bone marrow. Six of these seven grafts were lost between 6.7 and 25.5 months, whereas one was rejected early because of a humoral rejection in a highly sensitized patient in whom cytotoxic antibodies developed after surgery (Table 4). The non-rejection-related causes of pancreas loss in the two groups were not statistically different (see Table 4). Kaplan-Meier curves of kidney survival show an equal loss rate in both groups (Fig. 3). However, six of the eight kidney losses to rejection between 1 and 3.5 years were in the non-bone marrow group (Table 5).

The bone marrow and non-bone marrow groups were compared with reference to mycophenolate versus azathioprine use, and there was no difference in either pancreas or kidney survival (Figs. 4 and 5. respectively). Similarly, Figure 6 compares the pancreas survival curves according to the method of exocrine drainage; there was no difference, although only 19 of the 151 patients were bladder-drained.

The cumulative risk of rejection. comparing the bone marrow group with the non-bone marrow group. shows a striking difference (Fig. 7). The increase in the number of rejection episodes. including multiple rejections, in the nonbone marrow group compared with the bone marrow group is shown in Table 6. Forty-three percent of the bone marrow group but only $21 \%$ of the non-bone marrow group was rejection-free $(p=0.05)$. Further. twice as many patients in the non-bone marrow group had two or more rejections compared with the bone marrow recipients. and the number of rejections per patient was greater in the non-bone marrow group $(p=0.03$. see Table 6$)$.

Steroid withdrawal was more common in the bone mar- row group at 3 years after transplantation $(p=0.02$, Table 7). Patients were withdrawn from steroids based on their immunologic status. The non-bone marrow recipients had a mean tacrolimus level greater than that in the bone marrow group $(p=0.009$ ); this is not surprising, given that more rejection episodes occurred in the non-bone marrow group (Table 8). Further, the bone marrow patients appeared to have better pancreatic graft function, as reflected by a lower glycosylated hemoglobin value ( $\mathrm{p}=0.02$, see Table 8 ).

Causes of death in seven patients included four cardiacrelated, two sepsis, and one posttransplant lymphoproliferative disorder (PTLD; Table 9). The patient in the bone marrow group with posttransplant lymphoproliferative disease died with normal pancreatic and renal function. This patient was seronegative for Epstein-Barr virus, and the donor was seropositive. One patient in each group died from sepsis.

Bone marrow patients had a significantly greater $(92 \%)$ incidence of chimerism in their peripheral blood compared with the controls $(70 \%)$. This finding was consistent over the course of serial follow-up. Similarly, using semiquantitative polymerase chain reaction, the levels of donor cell chimerism were found to be at least two logs higher in the majority of the evaluated (male/female transplant recipients) bone marrow patients compared with the controls.

\section{DISCUSSION}

Results of pancreatic transplantation have improved steadily in the past 5 years. ${ }^{2}$ Tacrolimus-based immunosuppression has played a substantial role in this improvement, which was recently reported by Gruessner ${ }^{20}$ in a retrospective multicenter analysis. Our center began using tacrolimus from the inception of the program. and results, even in the early phase of the program. were far better than with conventional drug regimens. ${ }^{21}$ The surveillance strategy, in terms of frequency of fine-needle aspiration biopsies of both organs $^{22}$ and core kidney biopsies, resulted in the diagnosis of rejection with minimal, if any, abnormal laboratory findings. Further, appropriate use of tacrolimus, with high upfront dosing in the first few weeks. reduced the vigor of acute rejections in most cases and obviated the requirement

Table 8. LABORATORY VALUES AT RECENT FOLLOW-UP

\begin{tabular}{lccc}
\hline & $\begin{array}{c}\text { Bone Marrow } \\
\text { (Mean } \pm \text { SD) }\end{array}$ & $\begin{array}{c}\text { No Bone Marrow } \\
\text { (Mean } \pm \text { SD) }\end{array}$ & p Value \\
\hline Tacrolimus $(\mathrm{ng} / \mathrm{ml})$ & $8.9 \pm 2.6$ & $10.9 \pm 4.7$ & 0.009 \\
Hemoglobin $\mathrm{A}_{1 \mathrm{C}}$ & $5.07 \pm 0.8$ & $6.1 \pm 1.8$ & 0.02 \\
Creatinine $(\mathrm{mg} \%)$ & $1.3 \pm 0.4$ & $1.5 \pm 0.7$ & $\mathrm{NS}$ \\
Glucose $(\mathrm{mg} \%)$ & $96 \pm 27$ & $96 \pm 25$ & $\mathrm{NS}$ \\
Amylase $(\mathrm{U} / \mathrm{L})$ & $68 \pm 37$ & $75 \pm 62$ & $\mathrm{NS}$ \\
Lipase $(\mathrm{u} / \mathrm{L})$ & $113 \pm 109$ & $127 \pm 188$ & $\mathrm{NS}$ \\
& & & \\
\hline
\end{tabular}


Table 9. CAUSES OF DEATH IN 151 SPK PATIENTS

\begin{tabular}{|c|c|c|c|c|c|}
\hline \multirow[b]{2}{*}{ No. } & \multirow[b]{2}{*}{ Causes } & \multirow[b]{2}{*}{ Time $(\mathrm{mo})$} & \multirow[b]{2}{*}{ Bone Marrow } & \multicolumn{2}{|c|}{ Graft Function } \\
\hline & & & & Pancreas & Kidney \\
\hline$* 1$ & Lymphoma & 3.0 & Yes & Yes & Yes \\
\hline 2 & Sepsis & 5.7 & Yes & No & No \\
\hline 3 & Cardiac & 18.4 & No & No & Yes \\
\hline 4 & Sepsis & 21.8 & Yes & Yes & Yes \\
\hline 5 & Cardiac & 24.6 & Yes & No & Yes \\
\hline 6 & Cardiac & 38.4 & No & No & No \\
\hline 7 & Cardiac & 44.5 & Yes & No & No \\
\hline
\end{tabular}

for antilymphocyte induction. Although we were initially apprehensive regarding tacrolimus because of its reported hyperglycemic effect, ${ }^{23}$ when this did occur in a few patients, it was only transient and disappeared with reduction in the dose of tacrolimus and steroids. The long-term effect of tacrolimus on glucose metabolism. analyzed by Jordan et al, ${ }^{24}$ showed no deleterious effect in terms of abnormal fasting blood sugar values and glycosylated hemoglobin values.

Cytomegalovirus disease occurred in only one patient in this series, although the cytomegalovirus antigenemia (pp65) test was positive in 19 patients who were seronegative at the time of transplantation from a seropositive donor. The recipients who turned positive for cytomegalovirus antigenemia were treated preemptively with ganciclovir for 14 days, or until the antigenemia disappeared. The relative absence of cytomegaloviral disease was possibly a byproduct of avoiding antibody induction therapy.

Although we introduced the current method of bladder drainage more than a decade ago, ${ }^{17}$ we have returned to a modification of our original technique of enteric drainage $e^{25,26}$ to avoid the surgical complications related to bladder drainage. We performed 18 bladder-drained procedures in this series and reserved the bladder-drainage procedure for solitary pancreas transplants or pancreas after kidney transplants: the urinary amylase levels are useful in monitoring for rejection because the kidney cannot be used as a marker. In addition, we used bladder drainage when the graft is placed in the left iliac fossa to avoid performing the enteric anastomosis above the sigmoid colon or through its mesocolon. It is encouraging that only $2 / 37(6 \%)$ bladderdrained cases in our overall series of 177 patients required conversion to enteric drainage.

Although rejection of the allografted pancreas does occur in the bone marrow recipients. there were fewer episodes: more patients had no detectable rejection; more patients were weaned off steroids: better graft function existed. as measured by a lower glycosylated hemoglobin value: there was no pancreas loss to rejection after 3 months compared with six late rejection-related losses in the non-bone marrow recipients; and tacrolimus blood levels were lower.

The higher level of donor cell chimerism in the bone marrow recipients suggests that bone marrow administration augments the naturally occurring chimerism, which may shift the bidirectional immune responses to strengthen the graft-versus-host arm. Donor leukocytes have been shown to migrate to other organs and tissues in recipients after transplantation. ${ }^{27}$ These donor stem and dendritic cells continue to be immunologically active. Perhaps a partial tolerance is induced by clonal deletion or exhaustion, the phenomenon that occurs when $T$ cells are presented in a setting of excessive antigen, such as a pancreas/kidney/ duodenal organ transplant composed of lymphoid tissue in the duodenum and root of the mesentery. Immunosuppression is needed, particularly at the beginning, to establish engraftment and allow the bidirectional immunologic process to establish itself.

We reported a similar phenomenon in the fully allogeneic rat model with a pancreas/spleen transplant where the pancreas was accepted for the lifetime of the rat, provided that a short course of immunosuppression was administered at the outset. ${ }^{28}$ The same transplant experiment without transplanting the spleen, a virtual factory of pluripotent cells as well as other mature $T$ cells, resulted in graft loss to rejection in all cases despite the same course of immunosuppression. Although our previous experience with pancreatic and splenic transplantation in humans resulted in severe graftl'ersus-host disease, ${ }^{25.26}$ the permanent tolerance achieved in this allogeneic rat model suggests that there is a favorable bidirectional immune response.

In summary, bone marrow augmentation. in this clinical model. seemed to produce a beneticial effect from an immunologic point of view, at least in terms of reducing the incidence of rejection and late graft loss to rejection. In addition, these data suggest that tacrolimus-based immunosuppression without antilymphocyte induction is highly effective. Enteric drainage of the exocrine secretions appears to be an appropriate and perhaps preferable technique. In the 
future, more effective methods of achieving prolonged graft acceptance should be pursued, employing strategies to potentiate the beneficial effect of bone marrow augmentation on chimerism and graft acceptance.

\section{Acknowledgments}

The authors thank Lynn Ostrowski and Sheila Fedorek for data compilation.

\section{References}

1. Sollinger HW, Odorico JS, Knechtle SJ, et al. Experience with 500 simultaneous pancreas-kidney transplants. Ann Surg 1998; 228:284296.

2. Gruessner A, Sutherland DER. Analysis of United States and non-US pancreas transplants as reported to the International Pancreas Transplant Registry and to the United Network of Organ Sharing. In: Cecka JM, Terasaki PI, eds. Clinical transplants 1998. Los Angeles: UCLA Tissue Typing Laboratory; 1999:53-71.

3. Sugitani A. Gritsch HA. Shapiro R, et al. Surgical complications in 123 consecutive pancreas transplant recipients: comparison of bladder and enteric drainage. Transplant Proc 1998; 30:293-294.

4. Greussner RW, Sutherland DE. Troppmann C, et al. The surgical risk of pancreas transplantation in the cyclosporine era: an overview. J Am Coll Surg 1997; 185:128-144.

5. Zehr PS. Milde FK, Hart LK. Corry RJ. Life quality of pancreatic transplant recipients: a comparison. Transplant Proc 1992; 24:850851.

6. Corry RJ, Zehr P. Quality of life in diabetic recipients of kidney transplants is better with the addition of the pancreas. Clin Transplant 1990; 4:238-241.

7. Tyden G. Bolinder J. Solders G, et al. A 10-year prospective study of IDDM patients subjected to combined pancreas and kidney transplantation or kidney transplantation alone. Transplant Proc 1997; 29: 3119.

8. Fioretto P, Steffes NW, Sutherland DER, et al. Reversal of lesions of diabetic nephropathy after pancreas transplantation. $N$ Engl J Med 1998; 339(2):69-75.

9. Tyden G, Bolinder J, Tibell A, et al. Experience with single pancreatic transplantation in preuremic diabetic recipients in Stockholm. Transplant Proc 1992; 24:852-853.

10. Corry RJ. Egidi MF, Shapiro R. et al. Enteric drainage revisited. Transplant Proc 1995: 27:3048-3049.

11. Corry RJ. Egidi MF, Shapiro R. et al. Pancreas transplantation with enteric drainage under tacrolimus induction therapy. Transplant Proc 1997; 29:642.

12. Starzl TE. Demetris AJ, Murase N. et al. Cell migration, chimerism. and graft acceptance. Lancet 1992: 339:1579-1582.

13. Shapiro R, Starzl TE. Bone marrow augmentation in renal transplant recipients. Transplant Proc 1998: 30:1371-1374.

14. Rao AS. Shapiro R. Corry RJ, et al. Adjuvant bone marrow infusion in clinical organ recipients. Transplant Proc 1998: 30:1367-1368.

15. Kapur S. Bonham A. Dodson S. et al. Strategies to expand the donor pool for pancreas transplantation. Transplantation 1999: 67:284-290.

16. Pinna A. Dodson SF. Smith C. et al. Rapid en bloc technique for liver and pancreas procurement. Transplant Proc 1997: 29:647-648.

17. Nghiem DD. Corry RJ. Technique of simultaneous renal pancreaticoduodenal transplantation with urinary drainage of pancreas secretion. Am J Surg 1987: 153:4(05-4)6.

18. Fontes PS. Rao AS. Demetris AJ. et al. Bone marrow augmentation of donor-cell chimerism in kidney, liver, heart, and pancreas islet transplantation. Lancet 1994: 344:151-155.

19. Salgar S. Shapiro R. Dodson F. et al. Infusion of donor leukocytes to induce tolerance in organ allogratt recipients. J Leukocyte Biol 1999 (in press).
20. Gruessner RWG. Tacrolimus in pancreas transplantation: a multicenter analysis. Clin Trans 1997: 11:299-312.

21. Corry RJ, Shapiro R, Egidi MF, et al. Pancreas transplantation without antibody induction therapy. Transplant Proc 1998; 30:299-300.

22. Egidi MF, Shapiro R. Khanna A. et al. Fine needle aspiration biopsy in pancreatic transplantation. Transplant Proc 1995; 27:3055-3056.

23. Scantlebury V. Shapiro R. Fung JJ, et al. New onset of diabetes in FK 506- vs. cyclosporine-treated kidney transplant recipients. Transplant Proc 1991; 23:3169-3170.

24. Jordan ML. Shapiro R, Gritsch H, et al. Long-term results of pancreas transplantation under tacrolimus immunosuppression. Transplantation 1999; 67:266-272.

25. Starzl TE, Iwatsuki S. Shaw BW Jr, et al. Pancreaticoduodenal transplantation in humans. Surg Gynecol Obstet 1984; 159:265-272.

26. Corry RJ. Nghiem DD. Schulak JA, et al. Surgical treatment of diabetic nephropathy with simultaneous pancreatic duodenal and renal transplantation. Surg Gynecol Obstet 1986; 162:547-555.

27. Starzl TE, Zinkernagel RM. Antigen localization and migration in immunity and tolerance. N Engl J Med 1998; 339:1905-1913.

28. Wakely E, Oberholser JH, Corry RJ. Elimination of acute GVHD and prolongation of rat pancreas allograft survival with DST, cyclosporine. and spleen transplantation. Transplantation 1990; 48:72-77.

\section{Discussion}

Dr. David E.R. Sutherland (Minneapolis, Minnesota): Dr. Corry, your overall results in simultaneous kidney/pancreas recipients since 1994 are very good, with 3-year kidney and pancreas graft survival rates of over $90 \%$ and $80 \%$, respectively.

Despite your disclaimer, it seems that the chronic rejection rate is almost the same in the two groups, with and without donor bone marrow. The main effect of bone marrow was on the acute rejection episode rate. Most rejection episodes occurred in the first 6 months, and there was a $20 \%$ difference at that time point. Thereafter, the curves remained parallel. The chronic rejection rate seemed to be low in both groups and I would like you to comment on that.

How did you diagnose the rejection episodes? At the University of Minnesota, we have used a more or less constant protocol since 1994, without bone marrow, giving antithymocyte globulin (ATG) for induction with Prograf (tacrolimus), CellCept, and prednisone for maintenance immunosuppression. We recently analyzed our results from 1994-1998 and the rejection episode rates in the simultaneous pancreas/kidney (SPK) and the pancreas after kidney (PAK) groups were $25 \%$ at 6 months in both, much lower than what you reported even in your bone marrow group. So it is possible that the ATG is more effective at reducing the incidence of rejection episodes than bone marrow. On the other hand, what proportion of your rejection episodes were diagnosed solely by biopsy and were not apparent on clinical grounds?

Also, did you differentiate the rejection episodes diagnosed in the pancreas or the kidney? Do you do protocol biopsies in both the pancreas and kidney, or just the kidney?

Pancreas transplants alone are being done at increasing numbers. and this is where the future of pancreas transplantation lies. but there is still a higher rejection episode rate. When we analyze our data from 1994-1998. the same protocols that resulted in only a $25 \%$ rejection episode rate by 6 months in the SPK and PAK groups were associated with a $60 \%$ rejection episode rate in the pancreas transplant alone group. in spite of using ATG. It seems that the PTA group may be the one that would have the most benetit from the addition of the bone marrow. Finally, on theoret- 
ical groups we might have expected bone marrow to increase the early rejection episode rate because of the large load of passenger leukocytes directly stimulating the recipients' immune system. Later, when the bimodality of graft versus host and host versus graft balance occur, the benefit of bone marrow would be seen long-term. Yet it seems just the opposite. I would like your comments on this paradox.

Presenter DR. Robert J. Corry (Pittsburgh, Pennsylvania): I think as I showed in the two slides there was no rejection in the bone marrow group after 3 months, either chronic or acute. The seven losses occurred in the non-bone marrow group from 4 months to 3 years, so I think there is some protection against chronic rejection in the bone marrow patients.

Now, the diagnosis of rejection is an important point. A lot of these rejections were based on protocol biopsy without any clinical or laboratory findings, and "borderline" rejection was treated. Therefore, we are not necessarily basing the diagnosis of rejection solely on clinical or laboratory grounds. The pancreas-only group, I agree, would be a good group to try the bone marrow on, since those patients seem to have an increased rejection rate.

Dr. Clyde F. Barker (Philadelphia, Pennsylvania): Dr. Corry has presented a series of pancreas transplants today with remarkably fine results. I think it is appropriate to say that Dr. Corry is one of a very small group of investigators, including David Sutherland and just a few others, who are responsible for bringing pancreatic transplantation to its present level. As he said, it should no longer be considered an experimental procedure. Considering that only a few years ago, pancreas transplants could be expected to have no better than a $30 \%$ to $40 \%$ 1-year graft survival while the success of kidney transplants was about twice that good, it is quite amazing that Dr. Corry is now able to report almost $90 \%$ 1-year graft survival.

Ironically, Dr. Corry's overall results are so good that it is almost impossible to distinguish the outcome of his experimental (bone marrow-treated) group from that of the control (non-bone marrow-treated) patients. Patient pancreas and kidney survival at 1 and 3 years are virtually the same in both groups.

However, the difference in incidence of rejection episodes in the two groups is quite impressive. Rejection episodes were $50 \%$ more common in the non-bone marrow group than in the bone marrowtreated patients. Even more suggestive that bone marrow helps is that none of the pancreas grafts in bone marrow-treated patients were lost to rejection after 3 months, while seven in the control group were rejected.

It is important also, that by 3 years posttransplant. Dr. Corry succeeded in completely withdrawing steroids in $67 \%$ of the bone marrow-treated patients. However. I would like to ask him whether in a given patient he is able to decide with certainty whether steroids can be withdrawn and if so when? Is it possible that in the control patients (those not treated with bone marrow) some of the seven graft losses could have been avoided by not withdrawing the steroids. since in that group $45 \%$ were withdrawn from steroids?

Dr. Corry, do you have sufficient contidence in the bone marrow treatment that immunosuppression might be withdrawn completely? Dr. Starzl has in the past suggested this possibility. I wonder if Dr. Corry is considering this with his pancreas transplant patients.

That treatment of allograft recipients with donor bone marrow would have a beneticial impact on outcome certainly makes sense.
Long before Dr. Starzl made the important observation of persistent microchimerism in his long-term human survivors of renal and hepatic allografts, studies done by Billingham. Brent, and $\mathrm{Me}$ dawar in neonatal mice, and by Tony Monaco in adult mice, indicated that chimerism promoted by administration of donor bone marrow transfusion led to tolerance of subsequent tissue allografts from the same donor.

Another aspect of Dr. Corry's study is particularly intriguing. This interests me because of our own recent studies by Drs. Uchikoski and Naji in our laboratories at the University of Pennsylvania in an animal model of autoimmune diabetes, the BB rat. In these animals, the chimerism resulting from pancreaticoduodenal transplantation alone (without bone marrow augmentation) is quite substantial. This chimerism has a beneficial effect on graft survival but this is ablated by pretransplant irradiation of the graft to get rid of the passenger leukocytes.

This protective influence interestingly is even more effective in preventing autoimmune damage to the transplanted islets than it is in preventing allogeneic rejection. Since several recent reports indicate that human islet and pancreatic transplants are susceptible to damage by autoimmunity as well as rejection, I wonder if Dr. Corry has considered the possibility that some of his grafts have been damaged by autoimmunity rather than by rejection. and whether the bone marrow may be helpful in preventing autoimmune damage. Graft biopsies might provide some insight to this question.

This study is a very good one. I congratulate Dr. Corry on his excellent results.

DR. CoRry: I think that you have made a very important point that perhaps we should be a little bit more conservative in withdrawing steroids from the non-bone marrow group. I don't know whether the seven losses from the bone marrow group had steroid withdrawal earlier, but I will have to look that up and I will let you know.

I don't know whether I would at this point remove immunosuppression totally. We have several patients who are out 3 years just on tacrolimus alone. That strategy has been tried by John Fung's group in the patients who have long-term surviving livers, and I think they have had some success with that.

I can t really answer the autoimmunity question. I am aware of your rat study. We did have an earlier rat study that you may be aware of where we did the pancreas/spleen transplant. Providing we abrogated GVH, we got long-term survival for the lifetime of the rat just by the addition of the spleen. But I really can't answer the autoimmunity problem in the biopsies. I don't think it existed, however.

Dr. Donald C. Dafoe (Palo Alto, California): Dr. Corry, I would like to add my congratulations to the other discussants' for your excellent series of pancreas transplants and this innovative clinical trial of donor bone marrow infusion. And thank you for the opportunity to review your manuscript. The patient graft survival that was presented in a difficult group of patients with end-stage diabetic nephropathy is a tribute to the experience of Dr. Corry and his group-careful recipient and donor selection. and certainly better immunosuppression.

As acute rejection is brought under control with our current combinations of new drugs, noting that most organ recipients do not experience acute rejection now, the focus must shift to the problems of chronic immunosuppression-associated insidious side 
effects as well as chronic rejection. Although 1-year graft survivals of $85 \%$ have been achieved, the chronic graft loss rate presents a curve that runs parallel to that of a decade ago due to chronic rejection.

The concept of chimerism, which simplistically is often envisioned as a dynamic equipoise between rejection and GVH (graft versus host), has been championed by Dr. Starzl, Miami, and the Alabama group as the approach to induce tolerance. Your study supports an association between donor bone marrow infusion and allograft acceptance, and it is a promising start. I have a number of questions.

This is not a randomized study, and as we all know, biases do creep in. The withdrawal of steroids in the donor bone marrow group may be more aggressive or follow-up may be more vigorous, leading to a self-fulfilling prophecy. My question is, do you plan randomization?

You mentioned in your manuscript that PCR for chimeric cells was carried out. I did not see those data. And I would like to ask about the percentage of donor cells in the periphery and whether you sampled other compartments such as the host bone marrow or the graft itself.

There are multiple variables in this kind of trial, the dose of the bone marrow, the timing, the cell type infused, and whether a depletive regimen is used. What strikes me is that we need better endpoints rather than patient and graft survival.

DR. CORRY: I will try to answer those quickly. The randomization was not done except by the willingness of the donor family to give permission for removal of vertebral bodies. When that was done, the recipients received the marrow. When it was not done, they didn't receive the marrow. So that was the only randomization. The patients were made aware of this study on evaluation and signed a permit.

We didn't always remember who got bone marrow and who didn't. We didn't know that until we looked up this data. We did not sample the bone marrow or the organs in the recipients of long-term surviving grafts.

Regarding the chimerism data, I will try to get that for you when I discuss a portion of this topic at the ASTS meeting. It was essentially two logs higher in the bone marrow than in the nonbone marrow group. There was chimerism in the non-bone marrow group, but not as high a level.

Dr. George E. Pierce (Kansas City, Kansas): I also compliment Dr. Corry for this important and interesting paper. Bone marrow can markedly prolong allograft survival in a number of different experimental models but it has been very difficult to demonstrate this benetit in humans. This is not surprising since, as Dr. Barker has indicated. the results at 1 year and even 3 years are so good with conventional immunosuppression, it would be difficult to detect a small incremental benefit due to the bone marrow.

In fact, in your study, Dr. Corry, the 1-year and 3-year pancreas and kidney graft survival rates, as Dr. Barker has indicated, are very similar for the two groups. So the evidence that bone marrow is of benefit in your patients rests largely upon the significant difference in rejection rates. But the definition of rejection is somewhat arbitrary, and the diagnosis of rejection can be quite subjective. So I wonder if you could elaborate a little bit more on your diagnosis of rejection. Was it based solely on histologic criteria? Did you use any functional criteria or a combination of criteria to make the diagnosis of rejection?

With longer follow-up, this may not be a critical point because, as we will hear tomorrow from the Minnesota group, it appears that the best predictor of chronic rejection is acute rejection, i.e. acute rejection is the best predictor of long-term graft loss. So perhaps with longer follow-up-that is, 5 years and beyond-we may see a more clear-cut benefit in the group that received bone marrow, if indeed, as you suggest, bone marrow decreased the incidence of acute rejection.

I would like you to comment further on Dr. Starzl's interesting hypothesis that attempts to explain long-term graft acceptance on the basis of balanced graft versus host and host versus graft reactions. I am especially interested if you have any insight into the nature of the intriguing graft versus host component of this twoway mechanism.

I appreciate the opportunity to discuss this important paper.

Dr. Corry: I agree with everything you said.

The diagnosis of rejection was not based solely on clinical or laboratory findings. There were protocol fine-needle biopsies of both organs, the kidney and the pancreas, in the first 2 years, and there were protocol biopsies of the kidney at various times. We had rejection diagnosed with creatinines at 1.0 when the lipase was rising a little bit and we biopsied the kidney and showed rejection.

With regard to the two-way paradigm. I would have to refer you to the article by Starzl and Zinkernagle. In a 1990 paper, we discussed this concept in a rat model where we mentioned this bidirectional response, where there was GVH on the one hand in the rat spleen model and rejection on the other. Providing we were able to eliminate clinical GVH, this dual immune response resulted in survival for the lifetime of the animal. which did not occur if you didn't put the spleen in. which is a source of many pluripotent stem cells. I would refer you to that paper and the paper by Starzl and Zinkernagle and some of the others that Starzl has written, particularly the New England Journal Christmas Eve 1998 Review article.

I would like to thank all the discussants for their remarks. 